TRANSACTIONS OF THE

AMERICAN MATHEMATICAL SOCIETY

Volume 359, Number 9, September 2007, Pages 4537-4556

S 0002-9947(07)04314-0

Article electronically published on April 17, 2007

\title{
NORMAL FORM THEORY FOR RELATIVE EQUILIBRIA AND RELATIVE PERIODIC SOLUTIONS
}

\author{
JEROEN S. W. LAMB AND IAN MELBOURNE
}

\begin{abstract}
We show that in the neighbourhood of relative equilibria and relative periodic solutions, coordinates can be chosen so that the equations of motion, in normal form, admit certain additional equivariance conditions up to arbitrarily high order.

In particular, normal forms for relative periodic solutions effectively reduce to normal forms for relative equilibria, enabling the calculation of the drift of solutions bifurcating from relative periodic solutions.
\end{abstract}

\section{INTRODUCTION}

Normal forms are an important tool in the local analysis, including local bifurcations, of dynamical systems in the neighbourhood of elementary solutions, such as equilibria and periodic solutions. The aim of normal form theory is to find local coordinates in terms of which a dynamical system near an elementary solution has a convenient (simplest) form.

In systems with symmetry, or equivariant dynamical systems, elementary solutions include relative equilibria and relative periodic solutions (namely, solutions that reduce to equilibria and periodic solutions respectively when the symmetry group is quotiented out). In this paper, we develop normal form theory in the context of local bifurcations from relative equilibria and relative periodic solutions.

The first result in this direction was due to Fiedler and Turaev 8 who considered bifurcations from relative equilibria. They expressed the structure of the normal form in terms of resonances, as in 1, 12. We explore an alternative characterisation, in terms of additional equivariance conditions as in Elphick et al. [6], which has certain advantages described below. In addition, our method generalises to the case of relative periodic solutions.

1.1. Normal form theory for equilibria. To put our results in context, let us briefly summarize the normal form theory for nonequivariant systems due to Elphick et al. [6]. They proved that near an equilibrium of a vector field, coordinates can be chosen such that up to any desired order the normal form vector field is equivariant

Received by the editors November 15, 2005.

2000 Mathematics Subject Classification. Primary 37G40, 37G05, 37G15, 37C55.

The first author would like to thank the UK Engineering and Physical Sciences Research Council (EPSRC), the Nuffield Foundation and the UK Royal Society for support during the course of this research.

The first and second authors would like to thank IMPA (Rio de Janeiro) for hospitality during a visit in which part of this work was done. 
with respect to the action of the Lie group $G_{L_{S}^{T}}$ where

$$
G_{A}=\overline{\{\exp (t A): t \in \mathbb{R}\}} .
$$

Here, $L_{S}^{T}$ denotes the transpose of the semisimple part of $L 1$

More precisely, Elphick et al. 6 proved:

Theorem 1.1 (Elphick et al). Consider the ODE $\dot{x}=f(x)$ where $f: X \rightarrow X$ is a smooth vector field defined on a finite-dimensional vector space $X$. Suppose that $f(0)=0$ and let $L=(d f)_{0}$. Fix an inner product on $X$ and define transposes with respect to this inner product. Then for any $m \geq 1$, there is a near-identity polynomial change of coordinates that transforms $f$ into the form

$$
f(x)=\tilde{f}(x)+o\left(|x|^{m}\right),
$$

where $\tilde{f}$ is a polynomial of order $m$ satisfying $\tilde{f}(0)=0,(d \tilde{f})_{0}=L$ such that the nonlinear part of $\tilde{f}$, i.e. $\tilde{f}_{1}=\tilde{f}-L$, is $G_{L_{S}^{T}} \times G_{L_{N}^{T}}$-equivariant:

$$
\tilde{f}_{1}(\gamma x)=\gamma \tilde{f}_{1}(x), \quad \text { for all } \gamma \in G_{L_{S}^{T}} \text { and all } \gamma \in G_{L_{N}^{T}} .
$$

Remark 1.2. (a) In practice, we choose the inner product on $X$ so that $L$ commutes with $L_{S}^{T}$ (choose coordinates so that $L$ is in Jordan normal form and take the standard inner product relative to these coordinates). Then the normal form

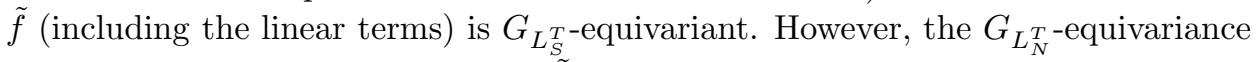
applies only to the nonlinear terms $\tilde{f}_{1}$.

(b) When focussing attention on the vector field restricted to the centre manifold, which is natural when studying local bifurcations, $G_{L_{S}^{T}}=G_{L_{S}}$ is a torus $T^{d}$ for some $d \geq 0$.

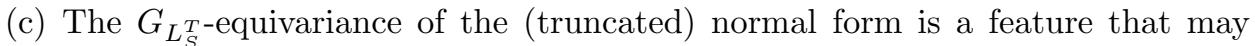
considerably aid the understanding of the local dynamics. At a more fundamental level, dynamical properties that are finitely determined, such as generic local branching patterns of equilibria and periodic solutions [9], may be crucially influenced by the normal form symmetry.

(d) In general, the normal form procedure does not converge, and terms in the tail (beyond all polynomial orders) may affect the qualitative dynamics (see for example [12, Chapter 7.4 and 7.5]).

A similar normal form theorem holds in the context of dynamical systems with symmetry. Let $\Gamma$ be a compact Lie group and suppose that $f$ is a $\Gamma$-equivariant vector field with a $\Gamma$-invariant equilibrium. Then there is a $\Gamma$-equivariant coordinate transformation such that the normal form $\tilde{f}$ is $\Gamma \times G_{L_{S}^{T}}$-equivariant 11]. Again, the normal form is characterised by the additional $G_{L_{S}^{T}} \times G_{L_{N}^{T}}$-equivariance in the nonlinear terms $\tilde{f}_{1}$, valid to arbitrarily high order.

We now describe the main results of our paper on normal forms for relative equilibria and relative periodic solutions.

\footnotetext{
${ }^{1}$ Recall that any linear operator $L$ on a finite dimensional vector space has a unique JordanChevalley decomposition into commuting semisimple and nilpotent parts: $L=L_{S}+L_{N}$ where $L_{S} L_{N}=L_{N} L_{S}$. The semisimple part $L_{S}$ is diagonalizable (over $\mathbb{C}$ ) and the nilpotent part $L_{N}$ satisfies the condition that $L_{N}^{p}=0$ for some $p[13$.
} 
1.2. Normal forms for relative equilibria. We consider bifurcations from a relative equilibrium for a smooth dynamical system $\dot{u}=F(u)$ satisfying the $\Gamma$ equivariance condition $F(\gamma u)=\gamma F(u)$ where $\Gamma$ is a finite-dimensional Lie group with Lie algebra $L \Gamma$. We denote the relative equilibrium by $u_{0}(t)=e^{t \eta} u_{0}$ where $\eta \in L \Gamma$. The isotropy subgroup of the relative equilibrium is given by

$$
\Delta=\left\{\gamma \in \Gamma: \gamma u_{0}=u_{0}\right\} .
$$

It is easy to see that $\delta u_{0}(t)=u_{0}(t)$ for all $\delta \in \Delta$ and $t \in \mathbb{R}$. We assume throughout that $\Delta$ is compact.

The dynamics in the neighbourhood of a relative equilibrium is governed by a skew product on $X \times \Gamma$ where $X$ is a $\Delta$-invariant slice transverse to the group orbit $\Gamma u_{0} ;$ see [7. The skew product equations take the form

$$
\dot{x}=f(x), \quad \dot{\gamma}=\gamma \xi(x),
$$

where $f: X \rightarrow X$ and $\xi: X \rightarrow L \Gamma$ satisfy the $\Delta$-equivariance conditions

$$
f(\delta x)=\delta f(x), \quad \xi(\delta x)=\operatorname{Ad}_{\delta} \xi(x),
$$

for $\delta \in \Delta$ and $x \in X$. (Here, $\operatorname{Ad}_{\delta} \xi=\delta \xi \delta^{-1}$.)

The structure of the equations expresses the fact that the equations are $\Gamma \times \Delta$ equivariant with respect to the action

$$
(x, \gamma) \mapsto\left(x, \gamma^{\prime} \gamma\right), \quad(x, \gamma) \mapsto\left(\delta x, \gamma \delta^{-1}\right),
$$

for $\left(\gamma^{\prime}, \delta\right) \in \Gamma \times \Delta$ and $(x, \gamma) \in X \times \Gamma$.

We assume that $f(0)=0$ and write $\xi(0)=\eta$. The underlying relative equilibrium $u_{0}(t)=e^{t \eta} u_{0}$ is thus identified with $(x, \gamma)(t)=\left(0, e^{t \eta}\right)$.

As a bifurcation parameter $\lambda$ is varied, and the relative equilibrium $u_{0}(t)$ may undergo bifurcations to new branches of solutions $u(t, \lambda)$. The drift dynamics along the group orbit is then governed by an equation of the form $\dot{\gamma}=\gamma \xi(x(t, \lambda), \lambda)$. If $\Gamma$ is abelian, we can solve this equation explicitly:

$$
\gamma(t)=\exp \left(\int_{0}^{t} \xi(x(s, \lambda), \lambda) d s\right) .
$$

However, for a general group $\Gamma$, the $\dot{\gamma}$ equation often cannot be solved explicitly. The simplest example is the case $\Gamma=\mathbf{S O}(3)$ studied by Wulff [21] and Comanici [5].

Fiedler and Turaev $[8$ approached this problem via normal form theory by simplifying the form of the drift equation beyond all orders. Chan [3] applied the normal form theory of Fiedler and Turaev to the case $\Gamma=\mathbf{S O}(3)$, greatly simplifying the calculations in [5, 21]. The method in [3] generalises to general compact groups (and certain noncompact groups) 4 .

The first result of this paper is a characterisation of normal forms in the neighbourhood of relative equilibria, in terms of additional equivariance conditions.

Theorem 1.3. Fix inner products on $X$ and $L \Gamma$. For any $m \geq 1$, there is a smooth $\Gamma$-equivariant near identity change of coordinates that transforms $f$ and $\xi$ into the form

$$
f(x)=\tilde{f}(x)+o\left(|x|^{m}\right), \quad \xi(x)=\tilde{\xi}(x)+o\left(|x|^{m}\right),
$$

where $\tilde{f}=L+\tilde{f}_{1}$ is a $\Delta$-equivariant polynomial of order $m$ satisfying the conditions in Theorem 1.1 and $\tilde{\xi}=\eta+\tilde{\xi}_{1}$ is a polynomial of order $m$ satisfying $\tilde{\xi}_{1}(0)=0$ and 
the $\Delta$-equivariance condition in (1.3), and

$$
\begin{aligned}
& \tilde{\xi}_{1} \circ \exp \left(t L_{S}^{T}\right)=\left(\operatorname{Ad}_{\exp (-t \eta)}\right)_{S}^{T} \tilde{\xi}_{1}, \\
& \tilde{\xi}_{1} \circ \exp \left(t L_{N}^{T}\right)=\left(\operatorname{Ad}_{\exp (-t \eta)}\right)_{U}^{T} \tilde{\xi}_{1},
\end{aligned}
$$

for all $t 2$

Moreover, the inner product on $L \Gamma$ can be chosen so that the equivariance condition in (1.5) is satisfied by the normal form $\tilde{\xi}$ (including the constant term).

This result is proved in Section 4. In general, the equivariance condition (1.6) for $\tilde{\xi}_{1}$ does not hold for the normal form $\tilde{\xi}$, though it does in fact apply to $\tilde{\xi}$ (for a suitably chosen inner product on $L \Gamma$ ) in many important special cases discussed in this paper, including the case when $\Gamma$ is compact. Even when (1.6) is not an equivariance condition for $\tilde{\xi}$, it may provide useful simplifications to the normal form.

Remark 1.4. Again (cf. Remark 1.2(d)), the normal form procedure does not converge in general, and terms in the tail may affect the qualitative dynamics.

In addition, the change of coordinates required for the normal form of $\tilde{\xi}$ mixes up the $x$ and $\gamma$ variables, and it is important to return to the original coordinates when interpreting the results. (For example, the phenomenon of meandering spirals, far from resonance, disappears beyond all orders in the normal form [8] but reappears at low order when transferring to the original coordinates.)

For the $\dot{\gamma}$ equation, it is often the second issue (returning to the original coordinates) that is more important than the first issue (terms beyond all orders); see for example [3]. This is in contrast to Birkhoff normal form theory for the $\dot{x}$-equation where the second issue is usually of no significance.

An important application of Theorem 1.3 concerns the computability of the drift in codimension-one local bifurcations from relative equilibria with trivial isotropy. We say that $\Gamma=K \ltimes \mathbb{R}^{n}$ is a Euclidean-type group if $\Gamma$ is the semidirect product of $\mathbb{R}^{n}$ with a compact Lie group $K$. This includes compact Lie groups $K$ as well as the Euclidean group $\mathbf{S E}(n)=\mathbf{S O}(n) \ltimes \mathbb{R}^{n}$.

Theorem 1.5. Suppose that $\Gamma$ is a Euclidean-type group. Then in a codimensionone bifurcation from a relative equilibrium with trivial isotropy $(\Delta=\mathbf{1})$, the group equation $\dot{\gamma}=\gamma \xi(x)$ is explicitly solvable in normal form.

The proof that this result follows from Theorem 1.3 is given in Section 2 , The same conclusion holds when all elements of the isotropy subgroup $\Delta$ of the relative equilibrium commute with all elements of $\Gamma$, i.e. if $\Delta \subset Z(\Gamma)$. Explicitly solvable means that the $\dot{\gamma}$ equation can be solved by repeated quadratures. In the compact case, this means that the normal form of $\xi(x)$ lies in an abelian subgroup so that the drift is given by (1.4). This generalises a result of Chan $[3$ for the group $\mathbf{S O}(3)$.

1.3. Normal forms for relative periodic solutions. We recall that by a result of Takens [20, the dynamics near a periodic solution can be described in normal form by the flow of a vector field in a transverse slice, where the periodic solution is represented by an equilibrium of the slice vector field. In the case of relative

\footnotetext{
${ }^{2}$ For invertible linear operators $L$, we have the decomposition $L=L_{S} L_{U}$, where $L_{S}$ is semisimple, $L_{U}$ is unipotent (that is, $L_{U}-I$ is nilpotent), and $L_{S} L_{U}=L_{U} L_{S}$. This decomposition is related to the decomposition $L=L_{S}+L_{N}$ by $L_{U}=I+L_{S}^{-1} L_{N}$.
} 
periodic solutions, we show that the local dynamics in normal form is effectively described by a vector field near a relative equilibrium:

Theorem 1.6. The dynamics near a relative periodic solution admits a reduction in normal form (beyond all orders) to the dynamics for a normal form vector field near a relative equilibrium.

Remark 1.7. (a) The isotropy subgroup of the relative equilibrium in Theorem 1.6 is in general a finite cyclic extension $\Delta \rtimes \mathbb{Z}_{2 p}$ of the isotropy subgroup $\Delta$ of the relative periodic solution. Similarly, the normal form vector field is equivariant with respect to the enlarged symmetry group $\Gamma \times\left(\Delta \rtimes \mathbb{Z}_{2 p}\right)$.

(b) The normal form vector field can be regarded as a general $\Gamma \times\left(\Delta \rtimes \mathbb{Z}_{2 p}\right)$ equivariant vector field possessing a relative equilibrium with isotropy $\Delta \rtimes \mathbb{Z}_{2 p}$. Hence the normal form theory for dynamics near relative periodic solutions reduces to the normal form theory for dynamics near relative equilibria as required. In particular, Theorem 1.3 applies in this situation.

(c) An immediate consequence of this discussion together with Theorem 1.5 is that when $\Gamma$ is of Euclidean-type, in codimension-one bifurcations from relative periodic solutions with trivial isotropy $(\Delta=\mathbf{1})$, the group equation governing drift is explicitly solvable in normal form.

We refer to Section [6, and in particular Theorem 6.5. for a detailed discussion of this result.

A previous approach proposed by Lamb et al. 16] reduces the dynamics near a relative periodic solution to that of a periodic solution in the slice and subsequently - by (Takens) normal form theory [20] - to that of an equilibrium. The method there suffices for the analysis of the dynamics in the slice, whereas the approach developed in the current paper deals simultaneously also with the dynamics in the group directions. In particular, resonances and rates of growth in the drift along the group can be efficiently computed for bifurcations from relative periodic solutions.

1.4. Normal forms in the presence of additional structure. In applications, other structures may be present in addition to equivariance. The problem is to characterise normal forms in terms of further equivariance conditions, whilst maintaining the underlying structure. A positive answer can be obtained when the vector fields with the specified structure form a Lie algebra. In these cases, the coordinate transformations achieving the normal form can be taken from the corresponding Lie group, and hence are structure preserving. Examples include Hamiltonian (symplectic) and volume preserving vector fields. For details on the structure of the equations of motion near relative equilibria and relative periodic solutions in Hamiltonian systems, see [19, 23].

Our normal form theory also extends to the context of reversible systems, where there is a reversing symmetry $R$, such that $x(t)$ and $R x(-t)$ are both solutions of the system. In this case the normalizing transformation can be chosen to be $R$-equivariant, naturally preserving the $R$-reversibility of the system. Reversible dynamical systems arise in a variety of applications [17. See 18 for the structure of equations near reversible relative equilibria and reversible relative periodic solutions.

The remainder of this paper is organised as follows. Theorem 1.3 simplifies greatly when the group of symmetries is compact, and this simplification is stated 
in Section 2, Also, Theorem 1.5 is proved in Section 2, In Section 3, we state the normal form theorem of Elphick et al. [6] in a general setting. In Section [4, we prove our main result, Theorem 1.3. on normal forms near relative equilibria. In Section 5 we discuss special (and hence simplified) cases of this theorem, beyond the compact case. In Section [6, we extend our results to relative periodic solutions.

\section{Normal FORMS FOR RELATIVE EQUILIBRIA WITH COMPACT SYMMETRY GROUPS}

Theorem 1.3 simplifies significantly when the symmetry group $\Gamma$ is compact, so in this section we state the results in this simplified setting. We describe the normal form of the vector field after centre manifold reduction, so that the eigenvalues of $L=(d f)_{0}$ lie on the imaginary axis.

2.1. The case when $L$ is semisimple. We first describe the consequences of Theorem 1.3 on the functions $\tilde{\xi}$ and $\tilde{f}$ defining the transformed bundle equations in the case that $L$ is semisimple.

Theorem 2.1. Suppose that $L$ is semisimple with eigenvalues on the imaginary axis, and that $\Gamma$ is a compact Lie group. For any $m \geq 1$, there is a smooth $\Gamma$ equivariant near identity change of coordinates that transforms $f$ and $\xi$ into the form

$$
f(x)=\tilde{f}(x)+o\left(|x|^{m}\right), \quad \xi(x)=\tilde{\xi}(x)+o\left(|x|^{m}\right),
$$

where

$$
\xi(0)=\tilde{\xi}(0)=\eta, \quad f(0)=\tilde{f}(0)=0, \quad(d f)_{0}=(d \tilde{f})_{0}=L
$$

and

$$
\tilde{f} \circ \exp (t L)=\exp (t L) \tilde{f}, \quad \tilde{\xi} \circ \exp (t L)=\operatorname{Ad}_{\exp (-t \eta)} \tilde{\xi},
$$

for all $t \in \mathbb{R}$.

Proof. By the assumptions on $L$, we can choose an inner product on $X$ so that $L$ is skew-symmetric. Hence $L_{S}^{T}=-L$ and $L_{N}^{T}=0$.

Similarly, since $\Gamma$ is compact, we can choose an inner product on $L \Gamma$ so that $\operatorname{Ad}_{\exp \eta}$ is orthogonal for all $\eta \in L \Gamma$. Then $\left(\operatorname{Ad}_{\exp \eta}\right)_{S}^{T}=\left(\operatorname{Ad}_{\exp \eta}\right)^{-1}=\left(\operatorname{Ad}_{\exp (-\eta)}\right)$ and $\left(\operatorname{Ad}_{\exp \eta}\right)_{U}^{T}=I$ for all $\eta$. The result follows immediately from Theorems 1.1 and 1.3 .

Define the groups

$$
G_{\eta}=\overline{\{\exp (t \eta): t \in \mathbb{R}\}} \subset \Gamma, \quad G_{L}=\overline{\{\exp (t L): t \in \mathbb{R}\}} \subset \mathbf{G L}(X) .
$$

Each of $G_{\eta}$ and $G_{L}$ is a torus. The normal form symmetry of $f$ in (2.1) reduces immediately to the condition that $f$ is $G_{L}$-equivariant. In general, the normal form symmetry for $\xi$ is more complicated, though generically the situation simplifies greatly. To describe the generic situation, it is useful to recall that $\operatorname{ad}_{\eta}: L \Gamma \rightarrow L \Gamma$ is the linear map given by $\operatorname{ad}_{\eta} \xi=\eta \xi-\xi \eta$. We adopt the following notion of resonance from 8 .

Definition 2.2. The element $\eta$ is resonant if $\operatorname{ad}_{\eta}$ has a nonzero eigenvalue that can be written in the form $n_{1} \nu_{1}+\cdots+n_{k} \nu_{k}$ where $n_{1}, \ldots, n_{k}$ are integers and $\nu_{1}, \ldots, \nu_{k}$ are eigenvalues of $L$. Otherwise $\eta$ is nonresonant. 
Corollary 2.3. If $\Gamma$ is compact, $L$ is semisimple, and $\eta$ is nonresonant, then the normal form as presented in Theorem 2.1 is $\Delta \times G_{L} \times G_{\eta}$-equivariant with respect to the action

$$
\begin{aligned}
& (x, v) \mapsto\left(\delta x, \operatorname{Ad}_{\delta} v\right), \quad \delta \in \Delta, \\
& (x, v) \mapsto(g x, v), \quad g \in G_{L}, \\
& (x, v) \mapsto\left(x, \operatorname{Ad}_{h} v\right), \quad h \in G_{\eta},
\end{aligned}
$$

for $(x, v) \in X \times L \Gamma$.

In other words,

$$
\begin{aligned}
& \tilde{f}(\delta x)=\delta \tilde{f}(x), \quad \tilde{f}(g x)=g \tilde{f}(x), \\
& \tilde{\xi}(\delta x)=\operatorname{Ad}_{\delta} \tilde{\xi}(x), \quad \tilde{\xi}(g x)=\tilde{\xi}(x), \quad \operatorname{Ad}_{h} \tilde{\xi}(x)=\tilde{\xi}(x),
\end{aligned}
$$

for all $\delta \in \Delta, g \in G_{L}, h \in G_{\eta}$.

Corollary 2.4. If $\Gamma$ is a compact Lie group, in a codimension-one bifurcation from a relative equilibrium with trivial isotropy $(\Delta=\mathbf{1})$, the group equation $\dot{\gamma}=\gamma \xi(x)$ is explicitly solvable in normal form.

Proof. Generically, in the case of codimension-one bifurcations, $L$ is semisimple. Since $\Delta=\mathbf{1}$, there are no constraints on $\eta$, and so generically $\eta$ is nonresonant: if $L=0$ (in the case of steady-state bifurcation), then $\eta$ is automatically nonresonant and if $L$ has eigenvalues $\pm i \omega$ (in the case of Hopf bifurcation), then $\eta$ is resonant if and only if $n \omega$ is an eigenvalue of ad $\eta$ for some nonzero integer $n$. Hence, $\eta$ generates a maximal torus $T^{d}$ in $\Gamma$. The last condition in (2.2) implies that $\xi(x)$ commutes with $\eta$ for all $x$ and so $\xi(x) \in L T^{d}$ for all $x$. Hence in the $\dot{\gamma}$ equation, we have $\xi(x(t, \lambda), \lambda) \in L T^{d}$ for all $t, \lambda$. Since $T^{d}$ is abelian, we obtain the explicit solution (1.4).

Proof of Theorem 1.5. Write $\gamma=\left(\gamma_{1}, \gamma_{2}\right) \in K \times \mathbb{R}^{n}, \xi=\left(\xi_{1}, \xi_{2}\right) \in L K \times \mathbb{R}^{n}$. A calculation using the semidirect product structure of $\Gamma=K \ltimes \mathbb{R}^{n}$ shows that $\gamma \xi=\left(\gamma_{1} \xi_{1}, \gamma_{1} \xi_{2}\right)$. Hence the drift equation $\dot{\gamma}=\gamma \xi(t, \lambda)$ reduces to

$$
\dot{\gamma}_{1}=\gamma_{1} \xi_{1}(t, \lambda), \quad \dot{\gamma}_{2}=\gamma_{1} \xi_{2}(t, \lambda)
$$

By Corollary 2.4 generically we can solve the $\dot{\gamma}_{1}$ equation explicitly for $\gamma_{1}(t, \lambda)$. Then $\dot{\gamma}_{2}=g(t, \lambda)$ where $g(t, \lambda)=\gamma_{1}(t, \lambda) \xi_{2}(t, \lambda) \in \mathbb{R}^{n}$. Since $\mathbb{R}^{n}$ is abelian we can solve explicitly for $\gamma_{2}$.

To include the resonant cases, we recall that $\operatorname{Ad}: \Gamma \rightarrow \operatorname{Aut}(L \Gamma)$ defines a representation of $\Gamma$ on $L \Gamma$ and hence restricts to a representation of $G_{\eta}$ on $L \Gamma$. Define the torus

$$
H=\overline{\left\{\left(\exp (t L), \operatorname{Ad}_{\exp (-t \eta)}\right): t \in \mathbb{R}\right\}} \subset G_{L} \times \operatorname{Aut}(L \Gamma) .
$$

Write elements of $H$ as $h=\left(h_{1}, h_{2}\right) \in G_{L} \times \operatorname{Aut}(L \Gamma)$. Then the normal form of $(f, \xi)$ is $\Delta \times H$-equivariant with respect to the action

$$
\begin{aligned}
& (x, v) \mapsto\left(\delta x, \operatorname{Ad}_{\delta} v\right), \quad \delta \in \Delta, \\
& (x, v) \mapsto\left(h_{1} x, h_{2} v\right), \quad h=\left(h_{1}, h_{2}\right) \in H .
\end{aligned}
$$

It is easily verified that this reduces to conditions (2.2) in the nonresonant case. 
2.2. The case when $L$ is nonsemisimple. For general $L$, Theorem 1.3 still simplifies when $\Gamma$ is compact. As in the proof of Theorem 2.1 we choose inner products on $X$ and $L \Gamma$ so that $L_{S}^{T}=-L_{S}$ and $\operatorname{Ad}_{\exp \eta}$ is orthogonal for all $\eta \in L \Gamma$. We state the result, omitting the straightforward proof.

Theorem 2.5. Suppose that $\Gamma$ is a compact Lie group. For any $m \geq 1$, there is a smooth $\Gamma$-equivariant near identity change of coordinates that transforms $f$ and $\xi$ into the form

$$
f(x)=\tilde{f}(x)+o\left(|x|^{m}\right), \quad \xi(x)=\tilde{\xi}(x)+o\left(|x|^{m}\right),
$$

where

$$
f(0)=\tilde{f}(0)=0, \quad(d f)_{0}=(d \tilde{f})_{0}=L, \quad \xi(0)=\tilde{\xi}(0)=\eta
$$

and

$$
\begin{aligned}
& \tilde{f} \circ \exp \left(t L_{S}\right)=\exp \left(t L_{S}\right) \tilde{f}, \quad \tilde{\xi} \circ \exp \left(t L_{S}\right)=\operatorname{Ad}_{\exp (-t \eta)} \tilde{\xi}, \\
& \tilde{f}_{1} \circ \exp \left(t L_{N}^{T}\right)=\exp \left(t L_{N}^{T}\right) \tilde{f}_{1}, \quad \tilde{\xi} \circ \exp \left(t L_{N}^{T}\right)=\tilde{\xi},
\end{aligned}
$$

for all $t \in \mathbb{R}$, where $\tilde{f}_{1}=\tilde{f}-L$ denotes the nonlinear part of $\tilde{f}$.

The definition of resonance is the same as in the semisimple case; see Definition 2.2. Nonresonance again leads to simplifications: the normal form is $\Delta \times$ $G_{L_{S}} \times G_{\eta}$-equivariant as in Corollary 2.3 (with $L$ replaced by $L_{S}$ throughout). The nonlinear part $\tilde{f}_{1}$ of $\tilde{f}$ is additionally $G_{L_{N}^{T}}$-equivariant, while $\tilde{\xi}$ is $G_{L_{N}^{T}}$-invariant.

\section{Extension of a Result By Elphick et al.}

In this section, we prove an extension of a result by Elphick et al. [6] underlying the characterisation of normal forms in terms of equivariance conditions.

Let $X$ and $Y$ be finite dimensional vector spaces, and let $L: X \rightarrow X$ and $M: Y \rightarrow Y$ be linear maps. Let $\mathcal{P}_{m}$ denote the vector space of polynomials $P$ : $X \rightarrow Y$ that are homogeneous of order $m$. We define an operator $\Phi_{L, M}: \mathcal{P}_{m} \rightarrow \mathcal{P}_{m}$ given by

$$
\Phi_{L, M}(P)=(d P) L-M P .
$$

More precisely, $\Phi_{L, M}(P)(x)=(d P)_{x} L x-M P(x)$.

Lemma 3.1. Given inner products on $X$ and $Y$, there exists an inner product on $\mathcal{P}_{m}$ with the property that for any linear maps $L: X \rightarrow X$ and $M: Y \rightarrow Y$,

$$
\operatorname{ker} \Phi_{L, M}^{T}=\left\{P \in \mathcal{P}_{m}: P \circ \exp \left(t L^{T}\right)=\exp \left(t M^{T}\right) P \text { for all } t \in \mathbb{R}\right\} .
$$

Proof. We break the proof into two steps. First, we choose an inner product on $\mathcal{P}_{m}$ such that $\Phi_{L, M}^{T}=\Phi_{L^{T}, M^{T}}$. Second, we show that

$$
\operatorname{ker} \Phi_{L, M}=\left\{P \in \mathcal{P}_{m}: P \circ \exp (t L)=\exp (t M) P \text { for all } t \in \mathbb{R}\right\} .
$$

The result follows.

Choose coordinates $x_{1}, \ldots, x_{d}$ on $X$ such that the inner product on $X$ is the natural inner product (so $L^{T}$ is the matrix transpose in these coordinates). If $\alpha$ and $\beta$ are multi-indices with $|\alpha|=|\beta|=m$, and $v, w \in Y$, we define

$$
\left\langle\left\langle x^{\alpha} v, x^{\beta} w\right\rangle\right\rangle= \begin{cases}\alpha !\langle v, w\rangle_{Y}, & \text { if } \alpha=\beta, \\ 0, & \text { if } \alpha \neq \beta .\end{cases}
$$

This extends by linearity to an inner product on $\mathcal{P}_{m}$. A direct calculation shows that $\Phi_{L, M}^{T}=\Phi_{L^{T}, M^{T}}$, completing the first step. 
Now define $R(t)=e^{-t M} P \circ e^{t L}$. Then $P \circ \exp (t L) \equiv \exp (t M) P$ if and only if $R$ is constant. A direct calculation shows that

$$
R^{\prime}(t)(x)=e^{-t M} \Phi_{L, M}(P)(y), \quad \text { where } y=e^{t L} x,
$$

completing the second step.

The Jordan-Chevalley decomposition of a linear operator $A=A_{S}+A_{N}$ has the property that $\operatorname{ker} A=\operatorname{ker} A_{S} \cap \operatorname{ker} A_{N}$. This enables a refinement of Lemma 3.1.

Lemma 3.2. Assume the setup of Lemma 3.1 and let $L=L_{S}+L_{N}, M=M_{S}+M_{N}$ be the Jordan-Chevalley decompositions of $L$ and $M$.

Then $P \in \operatorname{ker} \Phi_{L, M}^{T}$ if and only if

$$
P \circ \exp \left(t L_{S}^{T}\right)=\exp \left(t M_{S}^{T}\right) P \quad \text { and } \quad P \circ \exp \left(t L_{N}^{T}\right)=\exp \left(t M_{N}^{T}\right) P \quad \text { for all } t \in \mathbb{R} .
$$

Proof. We use the Jordan-Chevalley decomposition $\Phi_{L, M}=\left(\Phi_{L, M}\right)_{S}+\left(\Phi_{L, M}\right)_{N}$ to write

$$
\operatorname{ker} \Phi_{L, M}^{T}=\operatorname{ker}\left(\Phi_{L, M}\right)_{S}^{T} \cap \operatorname{ker}\left(\Phi_{L, M}\right)_{N}^{T} .
$$

Also, we have the decomposition $\Phi_{L, M}=\Phi_{L_{S}, M_{S}}+\Phi_{L_{N}, M_{N}}$. We claim that (i) $\Phi_{L_{S}, M_{S}}$ is semisimple, (ii) $\Phi_{L_{N}, M_{N}}$ is nilpotent, and (iii) $\Phi_{L_{S}, M_{S}}$ commutes with $\Phi_{L_{N}, M_{N}}$. It then follows by uniqueness that

$$
\left(\Phi_{L, M}\right)_{S}=\Phi_{L_{S}, M_{S}}, \quad\left(\Phi_{L, M}\right)_{N}=\Phi_{L_{N}, M_{N}},
$$

and so

$$
\operatorname{ker} \Phi_{L, M}^{T}=\operatorname{ker}\left(\Phi_{L_{S}, M_{S}}\right)^{T} \cap \operatorname{ker}\left(\Phi_{L_{N}, M_{N}}\right)^{T} .
$$

The result follows from Lemma 3.1

It remains to verify the claim. Parts (ii) and (iii) follow by direct calculation, with (ii) making use of the fact that $d^{m+1} P=0$. To verify part (i), let $\left\{x_{1}, \ldots, x_{d}\right\}$ be a (complexified) basis for $X$ in which $L_{S}$ is diagonal, and $\left\{v_{1}, \ldots, v_{m}\right\}$ a basis in which $M_{S}$ is diagonal. A basis for $\mathcal{P}_{m}$ is given by $\left\{x^{\alpha} v_{j}:|\alpha|=m, j=1 \ldots, n\right\}$ where $x^{\alpha}=x_{1}^{\alpha_{1}} \cdots x_{d}^{\alpha_{d}}$ is multi-index notation with $|\alpha|=\alpha_{1}+\cdots+\alpha_{d}$. It is immediate that $\Phi_{L_{S}, M_{S}}$ is diagonal in these coordinates as required.

Remark 3.3. In Lemma 3.2, we can write

$$
\exp \left(t M_{S}^{T}\right)=(\exp (t M))_{S}^{T}, \quad \exp \left(t M_{N}^{T}\right)=(\exp (t M))_{U}^{T}
$$

and similarly with $M$ replaced by $L$.

Remark 3.4. The case $X=Y$ and $L=M$ is the one considered in [6].

Discrete version. Let $L: X \rightarrow X, M: Y \rightarrow Y$ be as before, but suppose in addition that $M$ is invertible. Consider the operator $\Psi_{L, M}: \mathcal{P}_{m} \rightarrow \mathcal{P}_{m}$ given by

$$
\Psi_{L, M}(P)=P-M^{-1} P L .
$$

Lemma 3.5. Given inner products on $X$ and $Y$, there exists an inner product on $\mathcal{P}_{m}$ with the property that if $L: X \rightarrow X$ and $M: Y \rightarrow Y$ are linear maps with $M$ invertible, then $P \in \operatorname{ker} \Psi_{L, M}^{T}$ if and only if

$$
P \circ L_{S}^{T}=M_{S}^{T} P \quad \text { and } \quad P \circ L_{N}^{T}=M_{N}^{T} P .
$$

Proof. This is similar to, but simpler than, the proof of Lemmas 3.1 and 3.2 , 
Equivariance and twisted equivariance. We conclude this section by generalising to the case where $L$ and $M$ are equivariant or twisted equivariant. Let $\Delta$ be a compact Lie group $\Delta$ acting linearly on $X$ and $Y$. By averaging the inner products, we may suppose that $\Delta$ acts orthogonally on $X$ and $Y$.

The linear map $L: X \rightarrow X$ is $\Delta$-equivariant if $L \delta=\delta L$ for all $\delta \in \Delta$. It is easily verified that $L_{S}$ and $L_{N}$ are $\Delta$-equivariant. Since $\Delta$ acts orthogonally, $L^{T}$ is $\Delta$-equivariant. Similar comments apply to $M: Y \rightarrow Y$.

Let $\mathcal{P}_{m}(\Delta)$ denote the subspace of $\mathcal{P}_{m}$ consisting of $\Delta$-equivariant polynomials $P$ that satisfy $P(\delta x)=\delta P(x)$ for all $\delta \in \Delta$. Then $\Phi_{L, M}$ restricts to an operator on $\mathcal{P}_{m}(\Delta)$. The proofs of Lemmas 3.1 , 3.2 and 3.5 go through immediately to the $\Delta$-equivariant context.

Next suppose that $\phi \in \operatorname{Aut}(\Delta)$ is a finite order automorphism of $\Delta$. Then $L: X \rightarrow X$ is $\Delta$-twisted equivariant if $L \delta=\phi(\delta) L$ for all $\delta \in \Delta$. Provided $L$ is nonsingular, it can be shown that $L_{S}$ and $L_{N}$ are twisted equivariant [14, 16]. Since $\Delta$ acts orthogonally, $L^{T}$ is twisted-equivariant with respect to the inverse automorphism $L^{T} \delta=\phi^{-1}(\delta) L^{T}$.

Provided $L: X \rightarrow X$ and $M: Y \rightarrow Y$ are twisted equivariant with respect to the same automorphism $\phi \in \operatorname{Aut}(\Delta)$, the operator $\Psi_{L, M}$ restricts to $\mathcal{P}_{m}(\Delta)$. The proof of Lemma 3.5 goes through immediately to the twisted-equivariant context.

\section{NORMAL FORMS FOR RELATIVE EQUilibria}

In this section, we consider the skew product equations (1.2) when $\Gamma$ is a general finite-dimensional Lie group. We continue to suppose that $\Delta$ is compact.

The idea is to simplify the form of the skew product equations (1.2) through arbitrarily high (but finite) order, by making changes of coordinates that preserve the structure of the equations. Specifically, we consider changes of coordinates of the form

$$
(x, \gamma) \mapsto\left(P_{f}(x), \gamma P_{\xi}(x)\right),
$$

where $P_{f}$ and $P_{\xi}$ are near identity functions of $x$. Such changes of coordinates are $\Gamma$-equivariant. Moreover, provided $P_{f}$ and $P_{\xi}$ satisfy the $\Delta$-equivariance conditions (1.3), then the $\Delta$-equivariance of $f$ and $\xi$ is maintained by the change of coordinates.

It is convenient to carry out the $P_{f}$ and $P_{\xi}$ changes of coordinates separately. In Subsection 4.1, we review Birkhoff normal form theory for $f$, using the approach of Elphick et al. from Section 3. In Subsection 4.2, we carry out for $\xi$ the normal form theory of Fiedler and Turaev $[8]$ in the same spirit.

4.1. Normal form for the slice vector field $f$. We recall for convenience certain aspects of normal form theory for the equation $\dot{x}=f(x)$. Write $L=(d f)_{0}$ and note that $L$ commutes with the action of $\Delta$ on $X$. In the notation of Section 3, with $L=M$ and $X=Y$, we consider the linear operator

$$
\Phi_{L}=\Phi_{L, L}(P)=L P-(d P) L
$$

defined on the space of $\mathcal{P}_{m}(\Delta)$-equivariant homogeneous polynomials $P: X \rightarrow X$ of order $m$. Given an inner product on $\mathcal{P}_{m}(\Delta)$, we define

$$
V_{m}=\left(\Im \Phi_{L}\right)^{\perp}=\operatorname{ker} \Phi_{L}^{T} .
$$

Then by conventional Birkhoff normal form theory (see for example [12]) there is a $\Delta$-equivariant smooth near identity change of coordinates that transforms $f$ into 
the normal form

$$
L+f_{2}+f_{3}+\cdots+f_{m}+o\left(|x|^{m}\right),
$$

where $f_{j} \in V_{j}$ for $j=2, \ldots, m$.

Proof of Theorem 1.1. Given an inner product on $X$, we define an inner product on $\mathcal{P}_{m}(\Delta)$ as in Section 3 so that $V_{m}$ is the subspace of $\Delta$-equivariant polynomials that are additionally $G_{L_{S}^{T}}$-equivariant and $G_{L_{N}^{T}}$-equivariant. Hence the nonlinear terms $f_{2}, f_{3}, \ldots$ can be transformed to have these equivariance properties through arbitrarily high order.

Remark 4.1. (a) As discussed in Remark 1.2, in applications to bifurcation theory, it is natural to assume (after centre manifold reduction) that the eigenvalues of $L$ lie on the imaginary axis. Then we can choose the inner product on $X$ so that $L_{S}^{T}=-L_{S}$ and hence $G_{L_{S}^{T}}=G_{L_{S}}=T^{d}$. It follows that the normal form of $f$ truncated at any finite order is $\Delta \times T^{d}$-equivariant. When nontrivial, $G_{L_{N}^{T}}$ is a noncompact connected abelian group with one topological generator, and hence is a copy of $\mathbb{R}$. If $L$ is nonsemisimple, then the nonlinear terms of the normal form are $\Delta \times T^{d} \times \mathbb{R}$-equivariant.

(b) We note that $G_{L}$ has a single topological generator which excludes the possibility $G_{L^{T}}=T^{d} \times \mathbb{R}$, so in general $G_{L^{T}} \neq G_{L_{S}} \times G_{L_{N}^{T}}$. However, the $G_{L^{T} \text {-equivariant }}$ polynomials on $X$ are the same as the $G_{L_{S}} \times G_{L_{N}^{T}}^{N}$-equivariant polynomials on $X$.

4.2. Fiedler-Turaev normal form theory for $\xi$. We suppose that $f$ has already been transformed into normal form as discussed in Section 4.1. We now obtain analogous simplifications for $\xi$. Recall that $\eta=\xi(0)$ and $L=(d f)_{0}$ commute with the given actions of $\Delta$ on $L \Gamma$ and $X$ (so $\operatorname{Ad}_{\delta} \eta=\eta$ and $L \delta=\delta L$ ). Let $\mathcal{P}_{m}(\Delta)$ denote the space of homogeneous polynomials $P: X \rightarrow L \Gamma$ of degree $m$ satisfying the equivariance condition $P(\delta x)=\operatorname{Ad}_{\delta} P(x)$. by

Taking $M=-\operatorname{ad} \eta$, we have the linear operator $\Phi_{L, M}: \mathcal{P}_{m}(\Delta) \rightarrow \mathcal{P}_{m}(\Delta)$ given

$$
\Phi_{L, M}(P)=(d P) L+\operatorname{ad}_{\eta} P .
$$

For each $m \geq 1$, let $V_{m}=\left(\Im \Phi_{L, M}\right)^{\perp}$ (for a given choice of inner product).

Lemma 4.2 (Fiedler and Turaev 8]). Consider the skew product equations (1.2) and let $\eta=\xi(0), L=(d f)_{0}$. For any $m \geq 0$, there exists a $\Delta$-equivariant polynomial $P: X \rightarrow L \Gamma$ of order $m$ such that the change of coordinates $\gamma \mapsto \gamma \exp P$ transforms $\xi$ to

$$
\eta+\xi_{1}+\xi_{2}+\cdots+\xi_{m}+o\left(|x|^{m}\right),
$$

where $\xi_{j} \in V_{j}$ for $j=1, \ldots, m$.

Proof. We argue inductively. Suppose that $\xi$ has been transformed through order $m-1$. We make the transformation $\gamma=\delta \exp \left(P_{m}(x)\right)$ where $P_{m}$ is a homogeneous polynomial of some fixed degree $m \geq 1$, and compute the change of coordinates modulo terms of order $m+1$ or higher.

Set $\gamma=\delta \exp P_{m}$. Then

$$
\xi=\gamma^{-1} \dot{\gamma}=\exp \left(-P_{m}\right) \delta^{-1}\left\{\dot{\delta} \exp P_{m}+\delta \exp P_{m} d P_{m} \dot{x}\right\}=\operatorname{Ad}_{\exp P_{m}}^{-1} \delta^{-1} \dot{\delta}+d P_{m} f,
$$

and hence

$$
\delta^{-1} \dot{\delta}=\operatorname{Ad}_{\exp P_{m}}\left\{\xi-d P_{m} f\right\}=\exp \left(\operatorname{ad}_{P_{m}}\right)\left\{\xi-d P_{m} f\right\} .
$$


Thus $\xi$ is transformed into $\xi^{\text {new }}=\exp \left(\operatorname{ad}_{P_{m}}\right)\left\{\xi-d P_{m} f\right\}$. In particular, if $\xi_{m}$ denotes the terms in $\xi$ that are homogeneous of order $m$, then

$$
\xi_{m}^{\text {new }}(x)=\xi_{m}(x)+\operatorname{ad}_{P_{m}(x)} \eta-\left(d P_{m}\right)_{x} L x=\xi_{m}(x)-\operatorname{ad}_{\eta} P_{m}(x)-\left(d P_{m}\right)_{x} L x .
$$

Hence $\xi_{m}^{\text {new }}=\xi_{m}-\Phi_{L, M} P_{m}$ as required.

4.3. Characterisation of the subspaces $V_{m}$. At this point, we diverge from the treatment in [8]. We require the following technical lemma.

Lemma 4.3. Let $\eta \in L \Gamma$. Then there exists an inner product on $L \Gamma$ such that $\left(\exp \operatorname{ad}_{\eta}\right)_{S}^{T} \eta=\eta$.

Proof. The result is straightforward if $L \Gamma=\operatorname{gl}(V)$ where $V$ is a finite-dimensional vector space, since $\left(\exp \operatorname{ad}_{\eta}\right)_{S}^{T}=\exp \operatorname{ad}_{\eta_{S}^{T}}$. In general, $\eta_{S}^{T}$ is not meaningful, so the idea is to use Ado's Theorem (see for example [10, Appendix E.2]) to reduce to the case $L \Gamma=\operatorname{gl}(V)$.

The details are as follows. By Ado's Theorem, we can embed $L \Gamma$ as a subalgebra of $\mathbf{g l}(V)$. In particular, $\eta \in L \Gamma \subset \operatorname{gl}(V)$. Hence $\eta_{S}^{T}$ is defined as an element of $\operatorname{gl}(V)$. Choose an inner product on $V$ so that $\eta$ commutes with $\eta_{S}^{T}$.

Let ad denote the adjoint action of $\operatorname{gl}(V)$ on itself. So $\tilde{a d}_{A} B=B A-A B$ for all $A, B \in \operatorname{gl}(V)$. Ado's Theorem guarantees that $\operatorname{ad}_{\eta}: L \Gamma \rightarrow L \Gamma$ is the restriction of $\tilde{\mathrm{ad}} \eta: \operatorname{gl}(V) \rightarrow \operatorname{gl}(V)$.

In particular, $\tilde{a d}_{\eta_{S}^{T}} \eta=0$ and so $\exp \tilde{a d}_{\eta_{S}^{T}} \eta=\eta$.

Define an inner product on $\operatorname{gl}(V)$ by $\langle A, B\rangle=\operatorname{tr} A B^{T}$ (using the inner product chosen on $V)$. This restricts to an inner product on $L \Gamma$. Since $\left(\exp \tilde{a d}_{\eta}\right)_{S}$ is semisimple, the transpose as a linear operator on $L \Gamma$ is the restriction of the transpose as a linear operator on $\mathbf{g l}(V)$. Moreover,

$$
\left(\exp \operatorname{ad}_{\eta}\right)_{S}^{T} \eta=\left(\exp \tilde{a d}_{\eta}\right)_{S}^{T} \eta=\eta
$$

as required.

Proof of Theorem 1.3. This is an application of Lemma 4.2 with the inner product on $\mathcal{P}_{m}(\Delta)$ chosen as in Section 3. The result for the nonconstant terms $\tilde{\xi}_{1}$ follows from Lemma 3.2 and Remark 3.3 with $Y=L \Gamma$ and $M=-\operatorname{ad}_{\eta}$. (We have used the identity $\exp \circ \mathrm{ad}=\mathrm{Ad} \circ \exp$.) In (1.5), the constant term $\eta$ is taken care of by Lemma 4.3 .

\section{Calculation of $\left(\operatorname{Ad}_{\exp t \eta}\right)_{S}^{T}$ And $\left(\operatorname{Ad}_{\exp t \eta}\right)_{U}^{T}$}

In Section 2 we described a simplified version of Theorem 1.3 for the case $\Gamma$ compact. In this section, we discuss simplifications under more general assumptions on $\Gamma$.

We have the group homomorphism $\operatorname{Ad}: \Gamma \rightarrow \operatorname{Aut}(L \Gamma)$. In particular, $\operatorname{Ad}_{\exp \eta}=$ $\exp \left(\operatorname{ad}_{\eta}\right)$ is an element of $\operatorname{Aut}(L \Gamma)$. Let $K(\eta) \subset \operatorname{Aut}(L \Gamma)$ denote the closure of the one-parameter subgroup generated by $\operatorname{ad}_{\eta}$ :

$$
K(\eta)=\overline{\left\{\operatorname{Ad}_{\exp (t \eta)}: t \in \mathbb{R}\right\}}
$$

We recall the following elementary results; cf. [2]. 
Proposition 5.1. (a) $K(\eta)$ is isomorphic to a torus $T^{p}$ or to a line $\mathbb{R}$.

(b) $K(\eta)$ is a torus if and only if $\operatorname{Ad}_{\exp \eta}$ is a semisimple matrix with all eigenvalues on the unit circle. Equivalently, $\mathrm{ad}_{\eta}$ is a semisimple matrix with all eigenvalues on the imaginary axis.

(c) If $\Gamma$ is compact, then $K(\eta)$ is a torus. More generally, if $\overline{\{\exp (t \eta): t \in \mathbb{R}\}}$ is a torus in $\Gamma$, then $K(\eta)$ is a torus.

(d) If $\Gamma$ is abelian, then $K(\eta)=1$. More generally, if $\exp \eta \in Z(\Gamma)$ (equivalently, $\eta \in Z(L \Gamma)$ ), then $K(\eta)=\mathbf{1}$.

When $K(\eta)$ is a torus, Theorem 1.3 simplifies in a similar fashion to the case when $\Gamma$ is compact (discussed in Section 2).

Theorem 5.2. Suppose that $K(\eta)$ is a torus.

For any $m \geq 1$, there is a smooth $\Gamma$-equivariant near identity change of coordinates that transforms $\xi$ into the form

$$
\xi(x)=\tilde{\xi}(x)+o\left(|x|^{m}\right),
$$

where $\tilde{\xi}: X \rightarrow L \Gamma$ is a polynomial of order $m$ satisfying $\tilde{\xi}(0)=\eta$ and the $\Delta$ equivariance condition (1.3), and moreover

$$
\tilde{\xi} \circ \exp \left(t L_{S}^{T}\right)=\operatorname{Ad}_{\exp (t \eta)} \tilde{\xi}, \quad \tilde{\xi} \circ \exp \left(t L_{N}^{T}\right)=\tilde{\xi},
$$

for all $t$.

Proof. Choose the inner product on $X$ so that $L$ commutes with $L_{S}^{T}$. Choose the inner product on $L \Gamma$ so that $K(\eta)$ acts orthogonally on $L \Gamma$. Then the equivariance conditions in (1.5) and (1.6) reduce to (5.1).

Remark 5.3. If all the eigenvalues of $\operatorname{Ad}_{\exp \eta}$ lie on the unit circle, then $\left(\operatorname{Ad}_{\exp \eta}\right)_{S}$ topologically generates a compact Lie group $\hat{K}(\eta) \subset \operatorname{Aut}(\Gamma)$ (even though $K(\eta)$ need not be compact). In this case, we can again simplify condition (1.5) to the form $\tilde{\xi} \circ \exp \left(t L_{S}^{T}\right)=\left(\operatorname{Ad}_{\exp (t \eta)}\right)_{S} \tilde{\xi}$. This is the situation for Euclidean-type groups. However condition (1.6) requires more work as discussed below.

If $K(\eta)=\mathbb{R}$, then the situation is more complicated. To make further progress, we use Ado's Theorem to view $L \Gamma$ as a subalgebra of the space of real $n \times n$ matrices $M_{n}$ with Lie bracket $[A, B]=A B-B A$. It then makes sense to speak of the Jordan-Chevalley decomposition $\eta=\eta_{S}+\eta_{N} \in M_{n}$ for $\eta \in L \Gamma$. Also, we can define $\eta^{T} \in M_{n}$ for $\eta \in L \Gamma$. We caution that in general $\eta_{S}, \eta_{N}$ and $\eta^{T}$ depend on the choice of embedding and need not lie in $L \Gamma$.

Definition 5.4. Given an embedding $L \Gamma \subset M_{n}$, we say that $\eta \in L \Gamma$ satisfies property (SN) if $\eta_{S}$ and $\eta_{N}$ lie in $L \Gamma$. We say that $\eta$ satisfies property $(\mathrm{T})$ if $\eta^{T} \in L \Gamma$. We say that $\eta$ satisfies property (SNT) if $\eta_{S}^{T}$ and $\eta_{N}^{T}$ lie in $L \Gamma$.

If $\Gamma$ is compact, then every $\eta \in L \Gamma$ satisfies (SNT). For semisimple groups, (SN) is automatic [10, Appendix C.2] and the decomposition $\eta=\eta_{S}+\eta_{N}$ is independent of the embedding. For the classical groups, we have (SNT).

If $\eta$ satisfies $(\mathrm{SN})$, then $(\exp \eta)_{S}^{T}=\left(\exp \eta_{S}\right)^{T}$ and $(\exp \eta)_{U}^{T}=\left(\exp \eta_{N}\right)^{T}$. The obvious modifications of these statements hold when $\eta$ satisfies (T) or (SNT) leading to appropriately modified versions of conditions (1.5) and (1.6). 
5.1. Euclidean-type groups. Properties (SN) and (T) both fail for Euclideantype groups $\Gamma=K \ltimes \mathbb{R}^{n}, K \subset \mathbf{O}(n)$, with the usual embedding in $M_{n+1}$ given by $(A, v) \leftrightarrow\left(\begin{array}{ll}A & v \\ 0 & 1\end{array}\right)$. The corresponding Lie algebra embedding is $(B, w) \leftrightarrow\left(\begin{array}{cc}B & w \\ 0 & 0\end{array}\right)$ where $B \subset L K$ lies in the space of $n \times n$ skew-symmetric matrices and $w \in \mathbb{R}^{n}$.

Property (SN) can be recovered by normalising $\eta=(B, w)$ using the adjoint action of $\mathbf{E}(n)$ so that $B w=0$. It is then easily verified that $\eta$ satisfies (SN) with $\eta_{S}=(B, 0)$ and $\eta_{N}=(0, w)$.

In particular, $\eta_{S}$ generates a compact subgroup of $\Gamma$ and so we have the normal form symmetry

$$
\tilde{\xi} \circ \exp \left(t L_{S}^{T}\right)=\operatorname{Ad}_{\left(e^{t B}, 0\right)} \tilde{\xi} .
$$

A convenient choice of inner product on $L \Gamma$ is $\langle\langle(B, w),(C, z)\rangle\rangle=\operatorname{tr} B C^{T}+\langle w, z\rangle$. It is easy to check that $\left(\operatorname{ad}_{\eta}\right)_{N}^{T}(C, z)=\left(\frac{1}{2}\left(w z^{T}-z w^{T}\right), 0\right)$, and iterating this operator gives the zero operator. Hence

$$
\tilde{\xi} \circ \exp \left(t L_{N}^{T}\right)=A(t) \tilde{\xi},
$$

where $A(t)(C, z)=\left(C+\frac{1}{2} t\left(w z^{T}-z w^{T}\right), z\right)$.

Local bifurcation with $\mathbf{E}(n)$ symmetry, $n$ even. Write $\eta=(B, w)$. Since $n$ is even, generically $\eta$ generates a maximal torus $T^{d} \subset \mathbf{E}(n)$ where $d=n / 2$. Hence, we are in the situation of Theorem 5.2 .

Generically $\eta$ is nonresonant (the eigenvalues of $\eta$ are not integer combinations of the eigenvalues of $L$ ). Define $G_{L_{S}^{T}}$ and $G_{L_{N}^{T}}$ as in Section 2, Define $G_{\eta}=T^{d}$. The normal forms of $f$ and $\xi$ are then $\Delta \times G_{L_{S}^{T}} \times G_{\eta^{-}}$-equivariant, and apart from the linear term of $f$, they are $G_{L_{N}^{T}}$-equivariant.

The $G_{\eta}$-equivariance leads to the conclusion that $\xi$ lies in the constant maximal torus $T^{d} \times\{0\} \subset \mathbf{O}(n) \ltimes \mathbb{R}^{n}$. In particular, the $\dot{\gamma}$-equation is solvable in the nonresonant situation.

Local bifurcation with $\mathbf{E}(n)$ symmetry, $n$ odd. When $\Gamma=\mathbf{E}(n)$ with $n$ odd, after normalising $\eta=(B, w)$ so that $B w=0$, it is typically the case that $w$ is nontrivial. We restrict to the nonresonant case and define $G_{L_{S}^{T}}, G_{L_{N}^{T}}, G_{\eta_{S}}$ and $G_{\eta_{N}}$ where $\eta_{S}=(B, 0)$ and $\eta_{N}=(0, w)$.

Generically $G_{\eta_{S}}=T^{d}$ where $d=(n-1) / 2$. The $G_{\eta_{S}}$-equivariance already leads to solvable drift equations, and there are no further restrictions from $G_{\eta_{N}}$. We note that when $G_{\eta_{S}}$ is not a maximal torus, the $\mathbf{S O}(n)$-drift equations need not be solvable but the $\mathbb{R}^{n}$-drift equations are simplified as a consequence of the $G_{\eta_{N}}$-equivariance.

\section{NORMAL FORMS FOR RELATIVE PERIODIC SOLUTIONS}

Recall that a solution $u(t)$ for a $\Gamma$-equivariant ordinary or partial differential equation is called a relative periodic solution if $u(t)$ is not a relative equilibrium and there exists a $T>0$ (least) such that $u(T) \in \Gamma u(0)$. We may rescale time so that $T=1$. We define the group of spatial symmetries

$$
\Delta=\{\gamma \in \Gamma: \gamma u(0)=u(0)\} .
$$

By construction, there is an element $\sigma \in \Gamma$ such that $u(1)=\sigma u(0)$. The element $\sigma$ is called a spatiotemporal generator.

The aim in this section is to reduce the dynamics near a relative periodic solution to the dynamics near a relative equilibrium (modulo exponentially small effects). 
We will assume throughout that $\Gamma$ is an algebraic group. Roughly speaking, the reduction is accomplished as follows. Let $X$ denote a $\Delta$-invariant slice transverse to the group orbit of trajectories $\Gamma\{u(t)\}$. First, we use ideas of Wulff et al. 22] and Lamb and Melbourne [15] to show that the dynamics in a neighborhood of the relative periodic solution is governed by a diffeomorphism $F: \Gamma \times X \rightarrow \Gamma \times X$ with certain $\Gamma \times \Delta$-twisted equivariance properties stemming from the spatiotemporal symmetry. The relative periodic solution corresponds to a relative fixed point for $F$. Second, we obtain the decomposition (valid to arbitrarily high order)

$$
F=A \circ \exp h,
$$

where $A$ and $\exp h$ are diffeomorphisms which preserve the relative fixed point and commute with each other. Moreover, $A$ has finite order and $h: \Gamma \times X \rightarrow L \Gamma \times X$ is a $\Gamma \times \Delta$-equivariant vector field with a relative equilibrium (as usual, $\exp h$ denotes the time-one map of the corresponding flow). The relative periodic solution $u(t)$ reduces to a relative equilibrium for $h$. Hence the dynamics in the vicinity of the relative periodic solution $u(t)$ reduces (modulo exponentially small effects) to the study of the dynamics in the vicinity of a relative equilibrium.

The normal form vector field $h$ can be characterised as being equivariant with respect to a certain finite cyclic extension of $\Gamma \times \Delta$ (incorporating both the commutativity with the diffeomorphism $A$ and also the twisted-equivariance properties of $F$ ). This extra structure will be clarified as the section continues.

In Subsection 6.1 we recall a result of Wulff et al. 22] whereby the $\Gamma$-equivariant flow near the underlying relative periodic solution is lifted to a $\Gamma \times\left(\Delta \rtimes \mathbb{Z}_{2 n}\right)$ equivariant skew-product flow on $\Gamma \times X \times S^{1}$. Here, $\Delta \rtimes \mathbb{Z}_{2 n}$ is a finite cyclic extension of $\Delta$ that encodes the spatiotemporal symmetry of the relative periodic solution. In Subsection 6.2, following Lamb and Melbourne [15, we construct the $\Gamma \times \Delta$-twisted equivariant diffeomorphism $F: \Gamma \times X \rightarrow \Gamma \times X$ (the so-called "first hit-pullback map"). In Subsection 6.3, we obtain the normal form decomposition for $F$. (We note that it is only in this final subsection that we lose information about exponentially small effects.)

6.1. Skew product formulation. We begin by recalling the skew-product model from Wulff et al. 22, for dynamics in the vicinity of a relative periodic solution.

We assume throughout that $\Gamma$ is an algebraic group. This assumption ensures that we can choose the spatiotemporal symmetry $\sigma$ to have the decomposition $\sigma=\alpha e^{\eta}$, where $\alpha \in \Gamma, \eta \in L \Gamma$ satisfy $\alpha^{m_{0}}=e$ for some finite $m_{0}, \operatorname{Ad}_{\delta}(\eta)=\eta$ for all $\delta \in \Delta$, and $\operatorname{Ad}_{\alpha}(\eta)=\eta$. In particular, $e^{\eta} \alpha=\alpha e^{\eta}$.

Let $\phi: \Delta \rightarrow \Delta$ be the automorphism

$$
\phi(\delta)=\sigma^{-1} \delta \sigma,
$$

and let $k$ denote the order of $\phi$. Then $k$ divides $m_{0}$. Define $2 n=\operatorname{lcm}\left(m_{0}, 2 k\right)$ and let $S^{1}=\mathbb{R} /(2 n \mathbb{Z})$. Then we consider the skew-product equations

$$
\dot{\gamma}=\gamma f_{\Gamma}(x, \theta), \quad \dot{x}=f_{X}(x, \theta), \quad \dot{\theta}=1,
$$

where $f_{\Gamma}: X \times S^{1} \rightarrow L \Gamma$ and $f_{X}: X \times S^{1} \rightarrow X$ are smooth vector fields satisfying $f_{\Gamma}(0, \theta)=\eta$ and $f_{X}(0, \theta)=0$ for all $\theta \in S^{1}$. These equations are $\Gamma \times\left(\Delta \rtimes \mathbb{Z}_{2 n}\right)$ equivariant, where $\Gamma$ acts by left multiplication on the $\Gamma$-component and the action of $\Delta \rtimes \mathbb{Z}_{2 n}$ is given by

$$
\delta(\gamma, x, \theta)=\left(\gamma \delta^{-1}, \delta x, \theta\right), \forall \delta \in \Delta, \text { and } S(\gamma, x, \theta)=\left(\gamma \alpha^{-1}, Q x, \theta+1\right) .
$$


Here, $S$ generates $\mathbb{Z}_{2 n}$, and $Q \in \mathbf{G L}(X)$ is of order (at most) $2 k$ satisfying $Q^{-1} \delta Q=$ $\phi(\delta)$ for all $\delta \in \Delta$.

Equations (6.1) have the solution $P(t)=\left(e^{t \eta}, 0, t\right)$ which is relative periodic with relative period 1 . Indeed $P(1)=(\sigma, S) \cdot P(0)$. In addition, $(e, \delta) \cdot P(t)=P(t)$ for all $t$. Hence $P(t)$ is a relative periodic solution with spatial symmetry $\Delta$ and spatiotemporal generator $(\sigma, S)$.

Note that $\Delta \rtimes \mathbb{Z}_{2 n}$ acts freely on $\Gamma \times X \times S^{1}$. By equivariance, the flow on $\Gamma \times X \times S^{1}$ induces a $\Gamma$-equivariant flow on the quotient manifold $\Gamma \times X \times S^{1} / \Delta \rtimes \mathbb{Z}_{2 n}$. The relative periodic solution for the quotient flow has spatial symmetry $\Delta$ and spatiotemporal symmetry generator $\sigma$. Moreover, every $\Gamma$-equivariant flow defined locally near such a relative periodic solution can be realised as a quotient flow in this way. Hence, it suffices to study equations with the skew-product structure described in this subsection.

6.2. First-hit-pullback map for relative periodic solutions. Consider the base point $(e, 0,0) \in \Gamma \times X \times S^{1}$, and the $\Gamma \times \Delta$-invariant cross sections $\Gamma \times X \times\{0\}$ and $\Gamma \times X \times\{1\}$. Let $g^{(1)}$ be the first hit map of the vector field corresponding to these cross sections (so $\left.g^{(1)}(e, 0,0)=\left(e^{\eta}, 0,1\right)\right)$. The symmetry $(\alpha, S) \in \Gamma \times$ $\left(\Delta \rtimes \mathbb{Z}_{2 n}\right)$ is also a mapping between these cross sections. Writing $\Gamma \times X$ instead of $\Gamma \times X \times\{0\}$, we can define the first-hit-pullback map [15]

$$
F=(\alpha, S)^{-1} \cdot g^{(1)}: \Gamma \times X \rightarrow \Gamma \times X .
$$

Note that $F(e, 0)=\left(e^{\eta}, 0\right)$.

Proposition 6.1. The diffeomorphism $F: \Gamma \times X \rightarrow \Gamma \times X$ takes the form

$$
F(\gamma, x)=\left(\alpha^{-1} \gamma F_{\Gamma}(x) \alpha, L F_{X}(x)\right),
$$

where $F_{\Gamma}: X \rightarrow \Gamma$ and $F_{X}: X \rightarrow X$ are general smooth maps satisfying the $\Delta$-equivariance conditions

$$
F_{\Gamma}(\delta x)=\delta F_{\Gamma}(x) \delta^{-1} \text { and } F_{X}(\delta x)=\delta F_{X}(x), \forall \delta \in \Delta,
$$

and $F_{\Gamma}(0)=e^{\eta}, F_{X}(0)=0,\left(d F_{X}\right)_{0}=\operatorname{Id}_{X}$.

The linear map $L: X \rightarrow X$ is nonsingular and $\Delta$-twisted equivariant: $L \delta=$ $\phi(\delta) L$.

Proof. We can write $g^{(1)}(\gamma, x)=\left(\gamma g_{\Gamma}(x), g_{X}(x)\right)$, where $g_{\Gamma}: X \rightarrow \Gamma$ and $g_{X}$ : $X \rightarrow X$ are smooth maps satisfying the appropriate $\Delta$-equivariance conditions and $g_{\Gamma}(0)=e^{\eta}, g_{X}(0)=0$. The result follows from the definition of $F$, with $L=Q^{-1}\left(d g_{X}\right)_{0}$.

Proposition 6.2. The diffeomorphism $F$ is $\Gamma \times \Delta$-twisted equivariant, in the sense that

$$
F \circ\left(\gamma^{\prime}, \delta\right)=\left(\phi\left(\gamma^{\prime}\right), \phi(\delta)\right) \circ F, \forall\left(\gamma^{\prime}, \delta\right) \in \Gamma \times \Delta,
$$

where $\phi\left(\gamma^{\prime}\right)=\alpha^{-1} \gamma^{\prime} \alpha\left(\right.$ and $\left.\phi(\delta)=\alpha^{-1} \delta \alpha\right)$. 
Proof. Let $(\gamma, x) \in \Gamma \times X$. Then

$$
\begin{aligned}
\left(\phi\left(\gamma^{\prime}\right), \phi(\delta)\right) F(\gamma, x) & =\left(\phi\left(\gamma^{\prime}\right), \phi(\delta)\right)\left(\alpha^{-1} \gamma F_{\Gamma}(x) \alpha, L F_{X}(x)\right) \\
& =\left(\phi\left(\gamma^{\prime}\right) \alpha^{-1} \gamma F_{\Gamma}(x) \alpha \phi(\delta)^{-1}, \phi(\delta) L F_{X}(x)\right) \\
& =\left(\alpha^{-1} \gamma^{\prime} \gamma F_{\Gamma}(x) \delta^{-1} \alpha, L \delta F_{X}(x)\right) \\
& =\left(\alpha^{-1} \gamma^{\prime} \gamma \delta^{-1} F_{\Gamma}(\delta x) \alpha, L F_{X}(\delta x)\right) \\
& =F\left(\gamma^{\prime} \gamma \delta^{-1}, \delta x\right)=F\left(\gamma^{\prime}, \delta\right)(\gamma, x),
\end{aligned}
$$

as required.

Define $A_{\alpha, L}: \Gamma \times X \rightarrow \Gamma \times X$ by

$$
A_{\alpha, L}(\gamma, x)=\left(\alpha^{-1} \gamma \alpha, L x\right) \text {. }
$$

Since $A$ is a special case of $F$ (with $F_{\Gamma} \equiv e$ and $F_{X}=\operatorname{Id}_{X}$ ) it is immediate that $A_{\alpha, L}$ is $\Gamma \times \Delta$-twisted equivariant. Consequently, we can write

$$
F=A_{\alpha, L} \tilde{F},
$$

where $\tilde{F}: \Gamma \times X \rightarrow \Gamma \times X$ is $\Gamma \times \Delta$-equivariant and $\tilde{F}(e, 0)=\left(e^{\eta}, 0\right),\left(d \tilde{F}_{X}\right)_{0}=\operatorname{Id}_{X}$.

6.3. Takens normal form for the first-hit-pullback map. Our procedure below to bring the diffeomorphism $F$ into normal form is similar in spirit to the one introduced by Takens 20] for periodic solutions.

Let $\tilde{F}_{m}$ denote the $m$-jet of $\tilde{F}$ at $(e, 0)$. Recall that the set of $m$-jets of diffeomorphisms forms a Lie group. Moreover, $\tilde{F}_{m}(e, 0)=\left(e^{\eta}, 0\right)$, so that $\tilde{F}_{m}$ is isotopic to the identity near $v=0$. Hence for all $v$ near $0, \tilde{F}_{m}$ lies in the image of the exponential operator on the associated Lie algebra of vector fields; see for example 20. In fact, there exists a unique $m$-jet $h_{m}: \Gamma \times X \rightarrow L \Gamma \times X$ in the associated Lie algebra of vector fields so that $\tilde{F}_{m}=\exp \left(h_{m}\right)_{m}$. At the level of $m$-jets, we have the decomposition

$$
F=A_{\alpha, L} \exp h
$$

By uniqueness, $h$ inherits the $\Gamma \times \Delta$-equivariance of $\tilde{F}$. That is,

$$
h(\gamma, x)=\left(\gamma h_{\Gamma}(x), h_{X}(x)\right),
$$

where

$$
h_{\Gamma}(\delta x)=\operatorname{Ad}_{\delta} h_{\Gamma}(x) \text { and } h_{X}(\delta x)=\delta h_{X}(x), \forall \delta \in \Delta .
$$

In addition, $h_{\Gamma}(0)=\eta, h_{X}(0)=0$, and $\left(d h_{X}\right)_{0}=0$.

The decomposition (6.2) is of little use unless the diffeomorphisms $A_{\alpha, L}$ and $\exp h$ commute with each other. We record the following result for subsequent use.

Proposition 6.3. Let $\exp \hat{h}=A_{\alpha, L}^{-1}(\exp h) A_{\alpha, L}$. Then

$$
\hat{h}_{\Gamma}=\operatorname{Ad}_{\alpha} h_{\Gamma} \circ L, \quad \hat{h}_{X}=L^{-1} h_{X} \circ L .
$$

Proof.

$$
\begin{aligned}
& A_{\alpha, L}^{-1}(\exp h) A_{\alpha, L}(\gamma, x)=A_{\alpha, L}^{-1} \exp h\left(\alpha^{-1} \gamma \alpha, L x\right) \\
& \quad=A_{\alpha, L}^{-1}\left(\alpha^{-1} \gamma \alpha \exp h_{\Gamma}(L x), \exp h_{X}(L x)\right)=\left(\gamma \alpha \exp h_{\Gamma}(L x) \alpha^{-1}, L^{-1} \exp h_{X}(L x)\right) \\
& \quad=\left(\gamma \operatorname{Ad}_{\alpha} \exp h_{\Gamma} \circ L(x), \exp L^{-1} h_{X} \circ L(x)\right) .
\end{aligned}
$$


Let $\mathcal{P}_{\Gamma, m}(\Delta)$ denote the space of homogeneous polynomials $P: X \rightarrow L \Gamma$ of degree $m$ satisfying the equivariance condition $P(\delta x)=\operatorname{Ad}_{\delta} P(x)$. Taking $M=$ $\operatorname{Ad}_{\alpha}^{-1}$, we have the linear operator $\Psi_{L, M}: \mathcal{P}_{\Gamma, m}(\Delta) \rightarrow \mathcal{P}_{\Gamma, m}(\Delta)$ given by

$$
\Psi_{L, M}(P)=P-M^{-1} P L .
$$

Similarly, let $\mathcal{P}_{X, m}(\Delta)$ denote the space of homogeneous polynomials $P: X \rightarrow X$ of degree $m$ satisfying the equivariance condition $P(\delta x)=\delta P(x)$. We have the linear operator $\Psi_{L, L}: \mathcal{P}_{X, m}(\Delta) \rightarrow \mathcal{P}_{X, m}(\Delta)$ given by

$$
\Psi_{L, L}(P)=P-L^{-1} P L .
$$

Let $V_{\Gamma, m}=\left(\Im \Phi_{L, M}\right)^{\perp}$ and $V_{X, m}=\left(\Im \Phi_{L, L}\right)^{\perp}$ (for given choices of the inner product).

Lemma 6.4. Consider the first-hit pullback map $F=A_{\alpha, L} \exp h: \Gamma \times X \rightarrow \Gamma \times X$. For any $m \geq 0$, there exists a $\Gamma \times \Delta$-equivariant polynomial map $P: \Gamma \times X \rightarrow L \Gamma \times X$ of order $m$ such that the change of coordinates $F \rightarrow \exp (-P) F \exp P$ transforms $h$ so that

$$
h_{\Gamma}=\eta+h_{\Gamma, 1}+\cdots+h_{\Gamma, m}+o\left(|x|^{m}\right), \quad h_{X}=h_{X, 2}+\cdots+h_{X, m}+o\left(|x|^{m}\right),
$$

where $h_{\Gamma, j} \in V_{\Gamma, j}$ and $h_{X, j} \in V_{X, j}$.

Proof. We argue inductively. Suppose that $P_{\Gamma}$ and $P_{X}$ are homogeneous polynomials of degree $m$ in $v$. Define $\exp \hat{P}=A_{\alpha, L}^{-1} \exp P A_{\alpha, L}$. Then on the level of $m$-jets we have the equality

$$
\begin{aligned}
\exp (-P) F \exp P & =\exp (-P) A_{\alpha, L} \exp h \exp P=A_{\alpha, L} \exp (-P) \exp h \exp P \\
& =A_{\alpha, L} \exp (h+P-\hat{P}) .
\end{aligned}
$$

It follows from Proposition 6.3 that

$$
\hat{P}_{\Gamma}=\operatorname{Ad}_{\alpha} P_{\Gamma} \circ L, \quad \hat{P}_{X}(x)=L^{-1} P_{X} \circ L .
$$

The result follows.

Note that $\operatorname{Ad}_{\alpha}$ is semisimple since $\alpha$ has finite order. We choose the inner products on $L \Gamma$ and $X$ so that $\operatorname{Ad}_{\alpha}^{T}=\operatorname{Ad}_{\alpha}^{-1}, L_{S}^{T}=L_{S}^{-1}$. In particular $\operatorname{Ad}_{\alpha}^{T} \eta=$ $\eta$. Choosing complements to $V_{\Gamma, j}$ and $V_{X, j}$ as in Lemma 3.5. it follows that to arbitrarily high order

$$
h_{\Gamma} \circ L_{S}^{-1}=\operatorname{Ad}_{\alpha} h_{\Gamma}, \quad h_{X} \circ L_{S}^{-1}=L_{S}^{-1} h_{X} .
$$

We can write $L=L_{S} e^{N}=e^{N} L_{S}$ where $N$ is nilpotent. Applying Proposition 6.3, we deduce that $\exp h$ commutes with $A_{\alpha, L_{S}}$. Writing $\exp h=A_{e, \exp N} \exp \tilde{h}$, we obtain the commuting decomposition $F=A_{\alpha, L_{S}} \exp \tilde{h}$.

By [16. Theorem 4.1], we can write $L_{S}=L_{0} e^{B}=e^{B} L_{0}$ where $L_{0}$ is twisted equivariant of finite order, $B$ is equivariant, and $B$ has no eigenvalues in $\pi i \mathbb{Q}-\{0\}$. As in [16, the eigenvalue restriction on $B$ guarantees that commutativity with $A_{\alpha, L_{S}}$ is equivalent to $A_{\alpha, L_{0}}$. Hence writing $\exp \tilde{h}=A_{e, \exp B} \exp \hat{h}$, we obtain the commuting decomposition $F=A_{\alpha, L_{0}} \exp \hat{h}$.

As in [16, we let $\ell$ denote the order of $L_{0}^{2 k}$. Define $2 p=\operatorname{lcm}\left(m_{0}, 2 k \ell\right)$. 
Theorem 6.5. For any $m \geq 1$, there is a smooth $\Gamma \times \Delta$-equivariant near identity change of coordinates $T$, so that the first-hit return map $F$ satisfies up to order $m$ in $|x|$,

$$
T F T^{-1}=A_{\alpha, L_{0}} \exp h=\exp h A_{\alpha, L_{0}},
$$

where $\exp h$ is the time-one map of a $\Gamma \times\left(\Delta \rtimes \mathbb{Z}_{2 p}\right)$-equivariant vector field $h$ describing the neighbourhood of a relative equilibrium of $a \Gamma \times \mathbb{Z}_{2 p}$-equivariant vector field with isotropy $\Delta \rtimes \mathbb{Z}_{2 p}$. Here, $\mathbb{Z}_{2 p}$ is generated by $\alpha(\gamma, x)=\left(\gamma \alpha^{-1}, L_{0}^{-1} x\right)$.

The normal form vector field $h=\left(\gamma h_{\Gamma}, h_{X}\right)$ satisfies $h_{\Gamma}(0)=\eta$ and $\left(d h_{X}\right)_{0}=B$, and may be taken to be in the normal form for relative equilibria as described in Section 4 .

Proof. Apart from being $\Gamma \times \Delta$-equivariant, $h$ is also equivariant with respect to the action of $\mathbb{Z}_{2 p}$ : since $\exp h A_{\alpha, L_{0}}=A_{\alpha, L_{0}} \exp h$, where $h=\left(\gamma h_{\Gamma}, h_{X}\right)$, we have $h_{\Gamma}\left(L_{0} x\right)=\operatorname{Ad}_{\alpha^{-1}} h_{\Gamma}(x)$, so that the relative equilibrium has isotropy $\Delta \rtimes \mathbb{Z}_{2 p} \subset$ $\Gamma \times\left(\Delta \rtimes \mathbb{Z}_{2 p}\right)$.

Remark 6.6. The factor of 2 in the integer $2 p$ can be omitted when $L^{k}$ is $\Delta$ equivariantly isotopic to the identity, which is the case when $L^{k}$ has no eigenvalues at -1 ; see [16, Remark 2.4(b)].

\section{REFERENCES}

1. V. I. Arnol'd. Geometrical methods in the theory of ordinary differential equations, second ed., Grundlehren der Mathematischen Wissenschaften [Fundamental Principles of Mathematical Sciences] 250, Springer-Verlag, New York, 1988. MR947141 (89h:58049)

2. P. Ashwin and I. Melbourne. Noncompact drift for relative equilibria and relative periodic orbits. Nonlinearity 10 (1997) 595-616. MR.1448578 (98e:58125)

3. D. Chan. Hopf bifurcations from relative equilibria in spherical geometry. J. Differential Equations 226 (2006) 118-134. MR2232432

4. D. Chan and I. Melbourne. A geometric characterisation of resonance in Hopf bifurcation from relative equilibria. Preprint, 2006.

5. A. Comanici. Transition from rotating waves to modulated rotating waves on the sphere. SIAM J. Applied Dynamical Systems 5 (2006) 759-782.

6. C. Elphick, E. Tirapegui, M. E. Brachet, P. Coullet and G. Iooss. A simple global characterization for normal forms of singular vector fields. Physica D 29 (1987) 95-127. MR923885 (90d:58111a)

7. B. Fiedler, B. Sandstede, A. Scheel and C. Wulff. Bifurcation from relative equilibria to noncompact group actions: Skew products, meanders, and drifts. Doc. Math. J. DMV 1 (1996) 479-505. MR1425301 (97k:58111)

8. B. Fiedler and D. V. Turaev. Normal forms, resonances, and meandering tip motions near relative equilibria of Euclidean group actions. Arch. Rational Mech. Anal. 145 (1998) 129-159. MR.1664546 (99m:58171)

9. M. J. Field. Symmetry Breaking for Compact Lie Groups. Memoirs of the Amer. Math. Soc. 574, Amer. Math. Soc., Providence, RI, 1996.

10. W. Fulton and J. Harris. Representation Theory. Grad. Texts in Math. 129, Springer, New York, 1991. MR1153249 (93a:20069)

11. M. Golubitsky, I. N. Stewart, and D. Schaeffer. Singularities and Groups in Bifurcation Theory, Vol. II. Appl. Math. Sci. 69, Springer, New York, 1988. MR950168 (89m:58038)

12. J. Guckenheimer and P. Holmes. Nonlinear Oscillations, Dynamical Systems, and Bifurcations of Vector Fields. Appl. Math. Sci. 42, Springer, New York, Heidelberg, Berlin, 1990. MR:1139515 (93e:58046)

13. J. E. Humphreys. Introduction to Lie algebras and representation theory. Graduate Texts in Mathematics 9, Springer-Verlag, New York, 1978. MR499562 (81b:17007)

14. J. S. W. Lamb. Local bifurcations in $k$-symmetric dynamical systems. Nonlinearity 9 (1996) 537-557. MR1384491 (97d:58172) 
15. J. S. W. Lamb and I. Melbourne. Bifurcation from discrete rotating waves. Arch. Rational Mech. Anal. 149 (1999) 229-270. MR1726677(2001h:37108)

16. J. S. W. Lamb, I. Melbourne and C. Wulff. Bifurcation from periodic solutions with spatiotemporal symmetry, including mode interactions and resonances. J. Differential Equations 191 (2003) 377-407. MR.1978383(2004h:37076)

17. J. S. W. Lamb and J. A. G. Roberts. Time-reversal symmetry in dynamical systems: A survey. Physica D 112 (1998) 1-39. MR 1605826 (99b:58174)

18. J. S. W. Lamb and C. Wulff. Reversible relative periodic orbits. J. Differential Equations 178 (2002) 60-100. MR:1878526 (2003f:37084)

19. M. Roberts, C. Wulff and J. S. W. Lamb. Hamiltonian systems near relative equilibria. J. Differential Equations 179 (2002) 562-604. MR1885680(2003b:37082)

20. F. Takens. Forced oscillations and bifurcations. Commun. Math. Inst. Univ. Utrecht 3 (1974) 1-59. MR.0478235 (57:17720)

21. C. Wulff. Transitions from relative equilibria to relative periodic orbits. Doc. Math. J. DMV 5 (2000) 227-274. MR 1758877(2001f:37128)

22. C. Wulff, J. S. W. Lamb, and I. Melbourne. Bifurcation from relative periodic solutions. Ergodic Theory Dynam. Systems 21 (2001) 605-635. MR1827120(2002f:37088)

23. C. Wulff and M. Roberts. Hamiltonian systems near relative periodic orbits. SIAM J. Appl. Dyn. Syst. 1 (2002) 1-43. MR1893733 (2003h:37084)

Department of Mathematics, Imperial College London, London SW7 2AZ, United KINGDOM

E-mail address: jeroen.lamb@imperial.ac.uk

Department of Mathematics and Statistics, University of Surrey, Guildford GU2 7XH, United Kingdom

E-mail address: ism@math.uh.edu 